\section{Inferior alveolar nerve preservation for hemimandibulectomy and bridging plate reconstruction in monostotic fibrous dysplasia case}

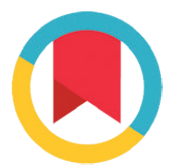

CrossMark

\section{Abstract}

Objective: This paper aimed to report the inferior alveolar nerve preservation in cases of monostotic fibrous dysplasia performed by hemimandibulectomy.

Methods: A 11-year-old boy was referred to Dr. Sardjito General Hospital with the complaint of having a lump at the lower back of the left jaw. Past medical history revealed that the patient fell and knocked a stone on the left jaw, at 8 years old. The patient was biopsied and diagnosed with monostotic fibrous dysplasia. From the result of the investigations conducted, the lesion had involved the left coronoid and condyle processes.
Results: The treatment performed for this case were hemimandibulectomy and reconstruction with installation of bridging plates to remove lesions, restore masticatory function and facial esthetics. Postoperative evaluation showed shape and jaw function was restored with no complaint of paresthesia.

Conclusion: Inferior alveolar nerve preservation was performed to minimize the symptoms of paresthesia as the patient was still young, the lesion was benign and had a low recurrence rate if it was taken cleanly.
Department of Oral and Maxillofacial Surgery, Faculty of Dentistry, Universitas Gadjah Mada, Yogyakarta, Indonesia
"Correspondence to: Agus Widodo, Department of Oral and Maxillofacial Surgery, Faculty of Dentistry, Universitas Gadjah Mada, Yogyakarta, Indonesia widodoagus06051981@gmail.com

Received: 15 February 2019 Revised: 10 July 2019

Accepted: 19 November 2019 Available Online: 1 December 2020

Keywords: Monostotic fibrous dysplasia, Hemimandibulectomy, Inferior alveolar nerve preservation, Bridging plate reconstruction Cite this Article: Widodo A, Rahmat MM, Prihartiningsih, Hasan CY. 2020. Inferior alveolar nerve preservation for hemimandibulectomy and bridging plate reconstruction in monostotic fibrous dysplasia case. Journal of Dentomaxillofacial Science 5(3): 196-199. D0l: 10.15562/jdmfs. v5i3.924

\section{Introduction}

Fibrous dysplasia is classified as a hamartoma or fibro-osseous lesion which is not a true neoplasm. The term dysplasia refers to a general disorder of tissue development, for example in the epithelium (epithelial dysplasia), mesenchymal tissue (fibrous dysplasia) or bony tissues (osseous dysplasia). ${ }^{1}$ Fibrous dysplasia is a benign, self-limiting, non-encapsulated lesion, often occurs in youth, more commonly affects the facial region of the mandible and involves a large portion of the bone. Radiographic features show normal bone turnover with fibrous tissue containing islands of trabecular metaplasia. $^{2}$

This disorder is generally diagnosed during growth, may affect only one bony structure (monostotic dysplasia) or multiple bones (polyostotic fibrous dysplasia). Bone fractures due to minor trauma are often associated with fibrous dysplasia abnormalities. Fractures caused by dysplasia of the bone is incapable of complete healing unless this fibrous tissue was treated surgically. The basic characteristic of fibrous dysplasia growth is self-limiting; during the initial phase, progressive damage to the bone occurs, then spread consistently and halt temporarily upon reaching skeletal maturation gradually, the metaplastic bone will be replaced by the lamellar bone but histologically, the tissue structures remain abnormal. The trabeculae of the lamellar bone is composed of thin and visible collagen fibers strung together like a shawl called lamelae arranged parallel to each other in the fibrous tissue stroma that remains. The bony structure at the final phase of fibrous dysplasia are often referred to as osseous keloid. ${ }^{3,4}$

Management of fibrous dysplasia depends on age, bone involvement and site of occurrence of abnormalities. If the lesion is small, periodic biopsy and evaluation would be carried out, however, if it had involved a large region of the mandible bone, the jaw must be removed. Radiotherapy of fibrous dysplasia is not recommended because it can potentially convert healthy cells into malignancies. ${ }^{2}$

\section{Case Report}

A 11-year-old male patient was referred to Dr. Sarjito General Hospital with the chief complaint of swelling in the left mandibular jaw. Past medical history revealed that the patient experienced a falling accident at the age of 8 years and knocked a stone on the left jaw. After the accident, there 

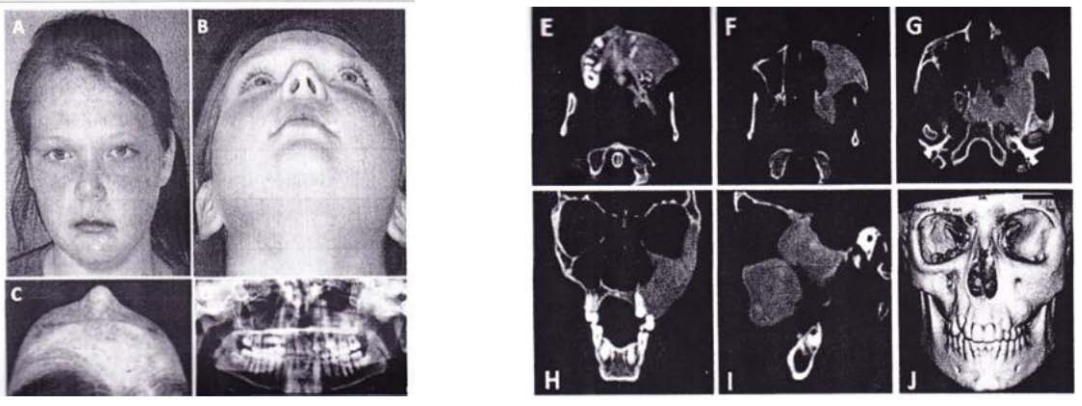

Figure 1 Monostotic fibrous dysplasia in a 11-year-old child. A-D. Clinical asymptomatic lesions, facial asymmetry and delayed dental eruption, E-J. CT scan shows a typical homogeneous picture of ground glass
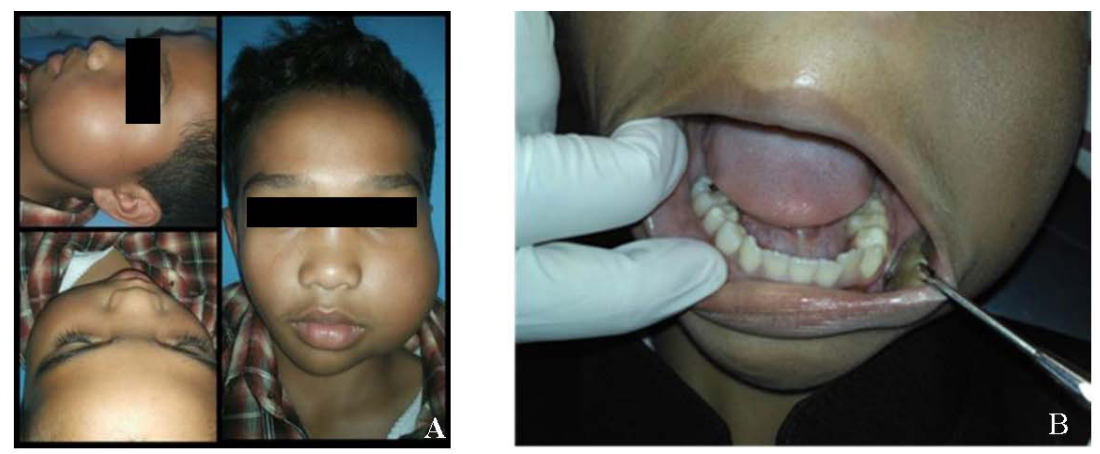

Figure 2 A. Extra oral clinical aspect, B. Intra oral clinical aspect
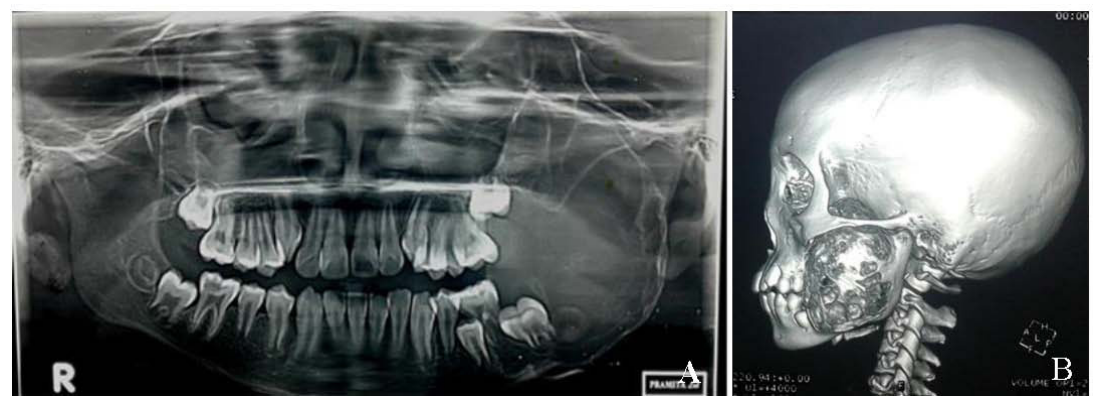

Figure 3 A. Orthopantomography, B. 3D CT-Scan
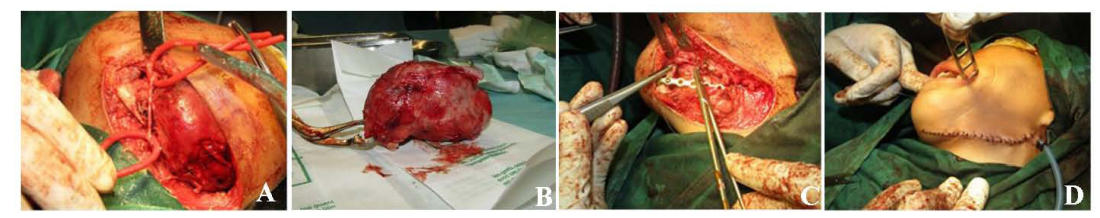

Figure 4 A. Preservation of the inferior alveolar nerve, B. After taking the lesion, C. Insertion of reconstruction plate, D. Suturing and drain installation

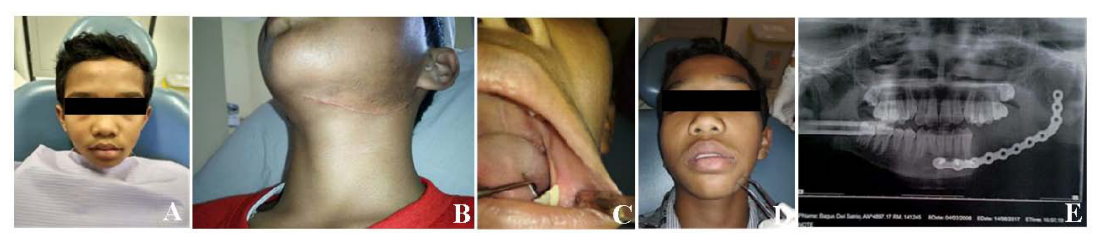

Figure 5 A. Clinical photo 8 months post op, B. Scar of the incision wound, C. Intra oral clinical photo, D. Pinprick test, E. 8 month postoperative OPG fracture, only mild bruises and swelling which later healed. A few months later there was a lump on the left jaw that grew larger.

Intra-oral examination showed the lumps in the buccal and lingual regions of 36 had extended posteriorly and superiorly towards the ramus and choroid. The lump was firm, hard, had a smooth surface, presented normal gingival color, and had no pain when palpated. Extra oral examination showed the lump extending superiorly (temporal) with a size of $8.5 \mathrm{~cm} \times 5.5 \mathrm{~cm}$. The patient had an asymmetrical face, while the lump was hard, presented no pain during palpation and had the same color as the surrounding tissue. The results of radiography and histopathology examination verified the diagnosis of monostotic fibrous dysplasia.

Hemimandibulectomy was performed, while maintaining (preservation) vital structures such as the inferior alveolar nerve and the left mental nerve, by releasing the nerves along the mandibular canal which had been pressed by the fibrous dysplasia mass towards the inferior mandible. Clearance of the nerves from the mental foramen was done by removing the bone with a bone bur directed towards posterior. In order to prevent damage to the nerve surface due to sharp objects or burs, the nerve was held using rubber (nelaton), inferior alveolar artery ligation, and the artery was severed in the mandibular foramen.

After insertion of the reconstruction plate, closure and restoration of the buccal and lingual periosteum attached to the bridging plate was performed, followed by suturing of the mucosa layer by layer using the horizontal mattress technique, until the plate was adequately covered by the muscles and mucosa. The inferior alveolar nerve was wrapped in the musculature and was not in contact with the bridging plate. The leather part was then sewn by interrupted suturing technique using 5-0 nylon cutting.

The results of the surgery performed on the patient was successful as there were no signs of infection and dehiscence in the operating area during the first week to 8 months postoperative evaluation. There were no signs of paresthesia but there were keloids in the former sutures. Evaluation was carried out for 8 months after hemimandibulectomy. During clinical examination of the inferior alveolar nerve at the beginning of postoperative evaluation, the patient had complaint of numbness in the lower left lip, hence the patient was given mecobalamin $250 \mathrm{mg} /$ 8 hours, placed under constant evaluation and paresthesia sensations disappeared after 1 month postoperatively. 


\section{Discussion}

Fibrous dysplasia is a bone abnormality that occurs during ages of rapid growth and development. Changes in bone structure occurs in the form of abnormal formation of mesenchymal tissue, where spongyose bony tissues are replaced with fibrous tissue. ${ }^{5}$ Fibrous dysplasia is a disorder of the bone where the bony tissues are replaced by abnormal fibrous tissue, resulting in asymmetric distortion and bone expansion. This can be confined to one bone type (monostotic fibrous dysplasia) or involve multiple bones (polyostotic fibrous dysplasia). Fibrous dysplasia is a benign fibroosseous lesion characterized with bony swelling that expands gradually in the maxilla and mandible. Monostotic type fibrous dysplasia often affects the jaws and the skull. $^{6}$

The FD lesions of the face may be described as quies-cent (stable with no growth), non-aggressive (slow grow-ing), or aggressive (rapid growth +/pain, paresthesia, pathologic fracture, malignant transformation, associa-tion with a secondary lesion). In the case of a quiescent FD lesion in which the patient does not complain of facial deformity, observation and monitoring for changes is an acceptable treatment modality. Annual evaluations may beadequate. The patient's concerns and symptoms, clinical assessment including sensory nerve testing in the region of involvement, photographs, and facial CT should be obtained at each visit.

Few examinations generally recommended in verifying the diagnosis of this case were incisional biopsy, bone surveys, and CT scans. Identification of newly formed lesions were carried out by bone survey procedure to determine the type of fibrous dysplasia. If polyostotic type lesions are found, consultation with an endocrinologist for endocrine and metabolic examination may be required, so that endocrine abnormalities could be identified as soon as possible for immediate diagnosis and treatment. In this case, results of bone survey in the patient did not indicate any other bone lesions. ${ }^{7}$

After mandibular resection, reconstruction plate was installed because hemimandibulectomy in young patients often causes extensive disability, and postoperative functional disorders. Mandibular reconstruction aimed to restore the shape and function of the face, maintain continuity and shape of the mandible and restore adequate muscular attachment, with the ultimate goal of improving the quality of life of patients. ${ }^{8,9}$

The inferior alveolar nerve is the main sensory nerve in the mandible which innervates the mucosa of the mouth, teeth and skin above the chin and lower lip. The inferior alveolar nerve is the third branch of the trigeminal nerve that extends from the base of the skull through the foramen ovale, after which it divides into the anterior and posterior stems. The inferior alveolar nerve appears inside the posterior stem and then passes through the mandibular foramen into the mandibular canal on the medial side of the mandibular ramus and across the canal, exiting the mental foramen. ${ }^{10}$

Nerves consist of axons and myelin that constitutes the nerve fibers. These fibers are encased in a thin layer of connective tissue called the endoneurium. A group of nerve fibers are joined together and encased by another layer of connective tissue, or perineurium, called fascicle. Some facies are arranged together and surrounded by a layer of connective tissue, known as the epineurium, thus forming the nerve bundles. ${ }^{10}$

The expansion of benign mandibular tumors associated with involvement of the inferior alveolar nerve canal had been widely reported. This also includes reports on shifting of the nerve canal and/ or destruction of the inferior alveolar canal and often with the displacement of the canal far from its normal position. Nerve involvement associated with tumors may cause alternate sensations in the patient (hypoaesthesia or hyperaesthesia). Because the inferior alveolar nerve travels inside the mandibular ramus, a large segment of the mandible body is usually resected with the tumor and occasionally may require mandibular removal in severe cases. Symptoms such as loss of nerve sensation, causing uncontrollable saliva, speech barriers, accidental biting of the lips, burning pain, lip tingling and paresthesia may result from mandibular resection surgery, hence management of the neurovascular bundle during tumor removal must be conducted cautiously. ${ }^{10}$

Many debates exist between oral and maxillofacial surgeons regarding the preservation of inferior alveolar neurovascular bundles during ablative surgery in the treatment of benign lesions of the mandible, especially in the management of ameloblastomas. An oral and maxillofacial surgeon, Becker reported in 1970, that maintenance of the inferior alveolar neurovascular bundle during mandibular tumor removal surgery is crucial, but this was not suggested for the treatment of multicystic ameloblastoma. Many oral surgeons recommend that this technique be used for benign cystic lesions such as cystic ameloblastoma and dentigerous cysts, as well as other benign pathologies including osteomyelitis of the mandible. ${ }^{12}$

Criterias for the preservation of mandibular alveolar nerves in benign lesions recommended 
by Ishikawa et al, 1986 and Hanlie, 2012 are: there should be no loss of sensation before surgery in the area of distribution of the inferior alveolar nerve, could be easily pulled, showing no signs of pathological adhesion, the nerve stem has normal elasticity thus minimizing injury, the nerve sheath must look smooth, shiny, uniform and regular.

In this case, the retrieval of fibrous dysplasia masses was performed by separating the tumor from the surrounding soft tissue. In a similar surgery conducted by Durante, the position of the mandibular canal and inferior alveolar nerve had been pushed inferiorly. In this case, the preservation of the nerve was carried out carefully from the mental foramen until the nerve was released to ensure both the inferior alveolar and mental nerve remains intact and unbroken without any effect on lip sensation. Loss in preservation of the alveolar nerve may result in a longer surgery time. ${ }^{13-15}$

\section{Conclusion}

Monostotic fibrous dysplasia in this case was a benign tumor of the oral cavity with a low recurrence rate and preservation of the inferior alveolar nerve was performed with satisfactory results after 8 months. The inferior alveolar nerve preservation in this case was aimed at reducing complaints associated with paresthesia after hemimandibulectomy and bridging plate insertion was conducted.

\section{Acknowledgment}

The authors have received permission from the patients depicted in the manuscript to use their photographs for this purpose.

\section{Conflict of Interest}

The authors report no conflict of interest.

\section{References}

1. Mansi A, Balaji N, Sumathi MK, et al. Fibrous dysplasia: a review. TMU J Dent 2014;1: 25-29.

2. Mohan H, Mittal P, Mundi I, et al. Fibrous dysplasia of bone: a clinicopathologic review. J Pathol Lab Med Int 2011;3: 1-42.

3. DiCaprio MR, Enneking WF. Fibrous dysplasia. pathophysiol, evaluation, and treatment. J Bone Joint Surg Am 2005;87: 1848-1864

4. Oluwaseun AA, Samuel ES, Vedant B, et al. Fibrous dysplasia: an overview of disease process, indications for surgical management and a case report. J ePlasty 2015;15: 37-46.

5. Kanasirska CRP. Fibrous dysplasia in the maxillomandibula region-case report. J IMAB-Ann Proceeding (Scientific Papers) 2010;16.

6. Mahadesh A. Fibrous dysplasia of the jaw bones. J Dent Sci \& Res 2011: 2-7.

7. Lee JS, FitzGibbon EJ, Chen YR, et al. Clinical guidelines for the management of craniofacial fibrous dysplasia. Orph J Rare Diseases 2012;7: 1-19.

8. Coen PD. Spontaneous bone regeneration after mandible resection in a case of ameloblastoma. Ann Acad Med Singapore 2004;33: 59-62.

9. Khodayari A, Khojasteh A, Kiani MT, et al. Spontaneous regeneration of the mandible after hemimandibulectomy: report of a case. J Dent Tehran 2011;8: 152-156.

10. Griffin MF, Malahias M, Hindocha S, et al. Peripheral nerve injury: principles for repair and regeneration. Open Orthop J 2014;8: 199-203.

11. Pourian A, Finkelstein M, Hellstein J. Ameloblastoma with involvement of theinferior alveolar neurovascular bundle: a case report. J Oral Surg Oral Med Oral Pathol Oral Radiol Endod 2011;1: 56-64.

12. Becker R. Continuity resection of the mandible with preservation of the mandibular nerve. $\mathrm{Br} \mathrm{J}$ Oral Maxillofac Surg 1970;8: 45-50.

13. Engelbrecht H, Meer S, Kourie JF. Perineural infiltration of the inferior alveolar nerve in mandibular ameloblastomas. Oral presentation. SASMFOS Congress 2011: 10-17.

14. Ishikhawa T, Noura N, Nagatha $\mathrm{H}$, et al. A new method of conserving the inferior alveolar nerve during resection of the mandible. British J Oral \& Maxillofac Surg 1986;24: 107-113.

15. Yongki R, Kawulusan NN, Purwanti I. Effectiveness comparison of inferior alveolar nerve block anesthesia using direct and indirect technique. J Dentomaxillofac Sci 2016;1: 171-176.

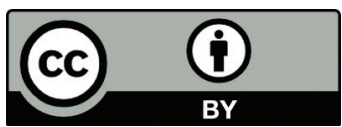

This work is licensed under a Creative Commons Attribution 